\title{
Urostomy Site Bleeding, CTCAE
}

National Cancer Institute

\section{Source}

National Cancer Institute. Urostomy Site Bleeding, CT CAE. NCI Thesaurus. Code C143912.

A disorder characterized by bleeding from the urostomy site. 